\title{
Implementation and Testing of Autonomous Quad-Rotor Aerial Vehicle by Using Ardu-Pilot
}

\author{
Rana Javed Masood, Wang Dao Bo, Zain Anwar Ali, Suhaib Masroor, and Muhammad Shafiq Loya
}

\begin{abstract}
In this article self-ruling autonomous quad-rotor, (AQR) Unmanned Aerial Vehicle (UAV)" system is developed. It has four rotors or engines and can fly without a pilot. The controlling and the velocity calculations of the UAV is control of diverse systems. The self - ruling UAV is accurately made to keep up harmony between velocity, weight, sound and force. It abides in four adjustable disperse arms with engines. Changes in engine velocities are utilized to adjust the position in the desired manner. All the association has been carried out in the middle of the UAV. Module is set inside the Ardupilot which is utilized to send signals to the engine with respect to the estimation of the sensors like GPS (U-Box GPS v2. 0), Gyro, Accelerometer and Barometer maintain a particular height. The venture is the implicit request to make a suitable and programmed flight starting in one direction, then in the next direction with defined height and pace. It must be remembered that the procurement of computations and investigation of the inertial measurement system must be performed precisely. Basically, this UAV full fill the needs of any clever gear, which is utilized to screen the ranges which are not in human reach.
\end{abstract}

Index Terms-Unmanned aerial vehicle, gyroscope, Ardu-Pilot.

\section{INTRODUCTION}

In the course of the most recent few years a rapid development in the production and offers on remote control airborne vehicles known as unmanned aerial vehicles (UAVs) are being used in several typical missions such as, search and rescue missions, surveillance, inspection, mapping, aerial cinematography and law enforcement. Many robust control schemes have been developed for the uncertain nonlinear systems. Among these control schemes, the sliding mode control, which has drawn researchers' much attention, has been a useful and efficient control technique for handling systems with large uncertainties, time varying properties, nonlinearities, and bounded external disturbances [1]-[4].

On the other hand, a conventional aircraft can travel faster, further and higher than a rotary wing. In order to merge these benefits on a single air vehicle, vertical takeoff, landing VTOL aircraft with a fixed wing configuration has been taken into consideration for over five decades by academicians and the industry itself [5], [6].

The quad - rotor UAV is the main concern in this paper. It has four arms and settled contribute propellers which are situated $\mathrm{X}$ or + setup with $\mathrm{X}$. Being the favored design. In the standard arrangement two propellers will turn in a clockwise direction with the other two turning in an anticlockwise course permitting to lift vertically, drift noticeable all around and fly in an assigned heading [7]. The AQR is a basic configuration with less moving parts and has quickly turned into a most loved vehicle for remote control fans and is generally being utilized as a viable Aerial photographic stage. An extensive larger part of the AQR was initially manufactured by specialists who comprehend the smoothness of the vehicle [8], [9].

It includes four engines and four propellers connected to a lightweight edge built of light wood, carbon fiber, or fiberglass then interface it to a remote control transmitter through a little control board fitted with a gyroscopic adjustment framework. Carrying out tests, the design of varieties of the AQR by utilizing distinct measures of arms seen Tri- Hex-Octa.

\section{SELECTION OF COMPONENT}

\section{A. Frame}

The essential and prominent component of AQR is its frame that supports engines and different hardware and escapes them from the vibrations. It is really precise when assembling.

\section{1) The arm}

Making of arm from materials like wood, Aluminum, Stainless Steel or PVC pipes, but it must validate the quality at the time of ruthless landings. The best weight of AQR frame is about 195 to 260 grams.

\section{2) Frame centre-plate}

It holds the arm of an AQR and different sensors lie on it. By utilization of glass fiber, wood ply, aluminum, or any material sheet they are lighter in weight.

\section{3) Size of frame}

Usually there is no fix frame size for moderate size AQR $435 \mathrm{~mm}$ to $560 \mathrm{~mm}$ is sufficient vary from engine to engine. The size of the frame is directly related to the energy like (Battery, ESC, Motor-proposition) includes a supplementary load. Using lobby king frame dimensions is given in the Table I.

TABLE I: FRAME SPECIFICATION

\begin{tabular}{|c|c|}
\hline Name & Size \\
\hline Engine length & $450 \mathrm{~mm}$ \\
\hline Height & $90 \mathrm{~mm}$ \\
\hline Width & $450 \mathrm{~mm}$ \\
\hline Weight & $170 \mathrm{~g}$ \\
\hline
\end{tabular}

Manuscript received October 30, 2015; revised January 13, 2016.

Rana Javed Masood, Wang Dao Bo, and Zain Anwar Ali are with Nanjing University of Aeronautics and Astronautics, China (e-mail: zainanwar86@hotmail.com).

Suhaib Masroor is with Shanghai University, China.

Muhammad Shafiq Loya is with the Department of Electronic Engineering, Sir Syed University of Engineering and Technology, Pakistan. 


\section{B. Engine}

The crucial part of AQR is engine, representing a major source of power consumption. In this research project we use the brushless DC motor for AQR. 600 to $1200 \mathrm{~V}$ rating engine are suitable for AQR. The Table II represents a necessary detail to choose the engine with respect to the required specification.

TABLE II: MOTOR SPECIFICATIONS

\begin{tabular}{ll}
\hline \hline Engine Essential Details & For Best Results \\
\hline KV & 935 \\
WEIGHT & $55 \mathrm{~g}$ \\
DIAMETER & $27.9 \mathrm{~mm}$ \\
LENGTH & $39 \mathrm{~mm}$ \\
BATTERY & $3 \mathrm{~s}(\mathrm{Li}-\mathrm{PO}) 10.45$ Prop \\
MAXIMUM THRUST & $850 \mathrm{~g}$ \\
ESCRECOMMENDED & $18 \mathrm{~A}$ \\
(For this Spec) & \\
\hline \hline
\end{tabular}

To elevate or lift about 1000 to 1200 grams AQR will need a cumulative thrust of about 2000 grams. AQR has four engines and every engine is supposed to generate thrust as a minimum as 500 grams to complete the requirement.

\section{1) Propellers}

Generally, ignore the piece of plastic in quad-rotor purpose assurance of propellers is amazing. Information of propellers is simple and they are spinning and pitch. The nature of the prop is crucial as well as the contrast of dimension and pitch on a flight of quad-rotor UAV. For the most part we see the prop with the determination of $7,3.5 ; 8,4.5 ; 9,5 ; 10,3.8 ; 10$, $4.5 ; 10,6 ; 11,4.7 ; 12,3.8$. (A span or Diameter in inches, Pitch in inches. Diameter: The Virtual circle that prop created).

Sum of movement / revolution seen over motor runs at 6660 RPM at no load. Anyway, after mounting the propellers on it, RPM will be diminished. Now taking the sample of two propellers 10, 3.8 and 10, 6. When the 10-inch measurement propeller mounts, the Revolutions per Minute (RPM) of the engine will be reduced to 3600 RPM. The foremost propeller is about 3.6-inches of pitch that grip all interruptions so $60 \times$ $3.8=216 \mathrm{Inch} / \mathrm{Sec}$ for the trice leg, it has a 6 -inch pitch $60 \times$ $6=360 \mathrm{Inch} / \mathrm{Sec}=9.1 \mathrm{~m} / \mathrm{Sec}[3]$.

In the event that $10,3.8$ prop our quad will move noticeable all around at 5.7 meters/second, while by 10, 6 prop Ascension rate will be increased to 9.1 meters/second. The large diameter propeller can create more push/thrust (Table III).

TABLE III: SYSTEM SPECIFICATIONS

\begin{tabular}{l}
\hline \hline SPECIFICATION's \\
\hline 6 DOF Acc-Gyro MPU 6000 \\
Barometer (Pressure Sensor) \\
Arduino Compatible \\
Open Source AP system \\
Atmel 's ATMEGA2560-16 au and ATMEGA32U \\
SPECIFICATION's \\
6 DOF Acc-Gyro MPU 6000 \\
Barometer (Pressure Sensor) \\
Arduino Compatible \\
Open Source AP system \\
Atmel 's ATMEGA2560-16 au and ATMEGA32U \\
\hline \hline
\end{tabular}

\section{2) Electronic speed controller}

The electronic speed controller is used to provide the constant power to the receiving side like servo-motor and the controller of flight. It has the Battery Eliminated Circuit (BEC) that requires only $5 \mathrm{~V}$. To choose better ESC for AQR, the current rating must be greater than the power rating of the motor.

\section{3) The battery}

Battery means power or fuel similar to a vehicle petrol tank. A filled tank will drive the vehicle more. So if we use high power rating battery in AQR it will fly more. Because AQR has four engines and in every engine consume $15 \mathrm{Amp}$ of power, thus the total requirement is about $60 \mathrm{Amp}$. Where "C" is the discharging rate of the battery. In this research project we use $40 \mathrm{C}$ battery because if we use $25 \mathrm{C}$ the calculation is about $2.2 \times 25 \mathrm{C}=55 \mathrm{~A}$ that is not enough for our $A Q R$ power that's why we use $40 \mathrm{C} \times 2.2=88 \mathrm{~A}$ which is useful for our AQR.

\section{4) The flight controller}

There are many controller boards; some of them are K.K Board, Multiwii Board, Naza Board. In this paper, we will discuss about the features of Ardu Mega Pilot, its specifications and modes.

\section{Movement of Autonomous QuAd-Rotor Vehicle}

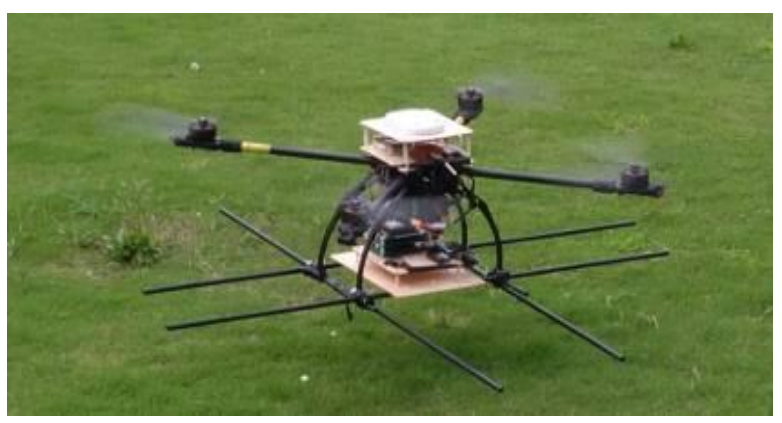

Fig. 1(a). Initial flight mode.

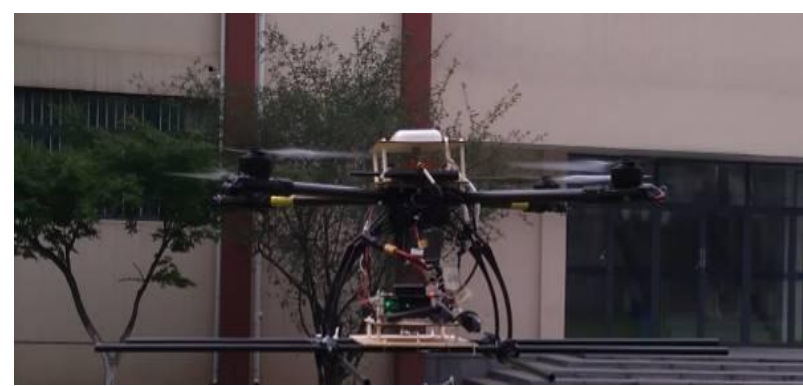

Fig. 1(b). Movement Flight mode.

An AQR acquired three Cartesian coordinates $(X, Y, Z)$ and 6-DOF via four control parameters. Dynamic inputs referring (Col, Lat, Lon, Ped.) are altitude, lateral, longitudinal and angular moments. $(p, q, r),(u, v, w)$ and $(\theta, \varphi$, $\psi)$ are the output of the vehicle and called as rotational velocities, translational velocities and rotational angles. Aerial robots exhibit a number of important physical effects such as aerodynamic effects, inertial counter torques, the gravity effect, gyroscopic effects and friction, etc. Due to these effects, it is difficult to design a real-time control for aerial robots. The UAV aerial robot is a highly nonlinear, multivariable, strongly coupled and under an actuated system 
since they have six degrees of freedom [4]. To organize the strategy of nonlinear sequential control to drive 6-DOF model, require rotational and translational subsystems. The movement of pitch, yaw and roll are as follows. Backward pitch, forward pitch, right roll, left roll, clockwise yaw, anticlockwise yaw, ascend and descend (see Fig. 1).

\section{TUNING PARAMETERS}

\section{A. Pitch Tuning}

Tuning the pitch and rate of roll gives most of the data. Stabilize Roll / Pitch P converts into the pivot rate at the desired point, which is then strengthened the rate of the controller. The higher the value makes copter more accessible to move/pitch, while a lower value makes it smoother. On the off chance that set excessively high, the copter will waver on the move and/or pitch pivot on the off chance that set excessively low the copter will get to be drowsy to inputs.

\section{B. Yaw Tuning}

Same like roll and pitch if any one stabilize, the yaw or yaw rate gets extremely higher and the AQR bearing or direction waver. At this time, the AQR will not be able to keep up its altitude.

\section{Altitude Tuning}

At the altitude hold tuning, the altitude " $P$ " by utilizing and changing the elevation error between the destination height and the real height the maximum rate produced will make an effort to keep up its elevation yet in the event that sets excessively high prompts an erratic throttle reaction. The rate of throttle does not require regular tuning or changes over the drop rate, but require a trip into a desired speed fall and down. The throttle accelerates the Proportional Integral Derivative (PID) controller additionally changing the speed (i.e. The contrast between the desired action and increasing its speed) into an engine yield. The 1:2 proportional ratio of $\mathrm{P}$ and $\mathrm{I}$ is twice the measuring of $\mathrm{P}$ has to be kept up of the event that regulates these three parameters. These qualities have never to be expanded yet for compelling the AQR may show signs of improvement and its reaction by decreasing its P \& I to 0.5:1.0 (see Fig. 2).

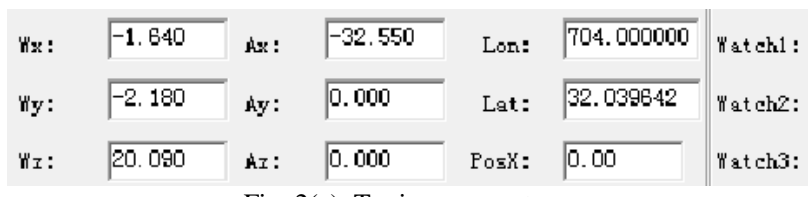

Fig. 2(a). Tuning parameters.

\begin{tabular}{|c|c|c|c|c|c|c|c|}
\hline Pitmo: & 0.000 & PLon: & -6.0 & PHM1: & 13266.000 & PosY: & 0.00 \\
\hline Rolבmo: & -0.160 & PLat: & 101.0 & PHIN2: & $\longdiv { 1 2 9 0 3 . 0 0 0 }$ & Pos $Z:$ & 0.00 \\
\hline 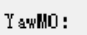 & 0.000 & FT:ail: & 307.0 & FWH3: & 13405.000 & SpdX: & -0.31 \\
\hline DiבMo: & 0.450 & POil: & 3309.0 & FHM4: & $\longdiv { 1 3 0 1 6 . 0 0 0 }$ & $\mathrm{s}_{\mathrm{p}} \mathrm{dY}:$ & 0.64 \\
\hline Tempe: & 20.09 & State: & 11 & GPS: & 12 & $\mathrm{~S}_{\mathrm{p}} \mathrm{dz}:$ & 0.14 \\
\hline
\end{tabular}

\section{Inner PID Controller}

PID circle look at the rate of precise transformation and complexities that with the crude gyro yield. The qualification is informed into the PID controller and sent to the motors to cure the upset. This is the heart of both rate modes; stabilize mode, and all distinctive modes. It is similarly the most fundamental expansion to alter the copter. The outside PI circle delivers the fancied rate of angular turn. The data for this circle can either be the customer with stick advancement, or the stabilizer, which tries to achieve a specific point. To enhance the surge of request to motor yield, here is a blueprint of roll control (see Fig. 3).

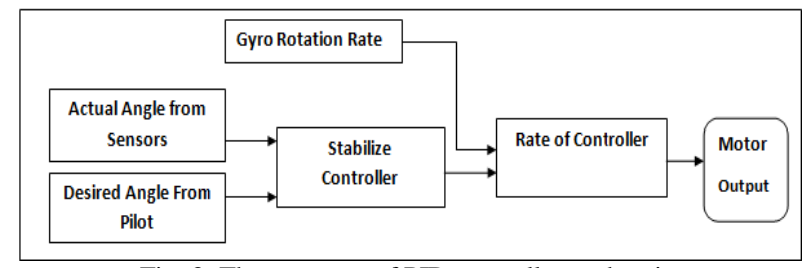

Fig. 3. The structure of PID controller each axis.

\section{MODES}

Following are the modes of operations in which AQR will operate.

\section{A. ALT Hold}

ALT hold means altitude hold or height hold at a particular point. As a result, the throttle controls the current elevation point. The tuning of pitch, yaw and roll is identical in stabilizing mode that's implying the particular control of pitch and roll and the direction.

\section{B. RTL}

(GPS Dependent) (RTL) mode, the AQR explores from its present position to float over the home position. The conduct of RTL mode can be controlled by a few movable parameters. At the point when RTL mode is chosen, the copter will come back to the home area. The copter will first climb to RTL_ALT before returning home or keep up the current height if the current elevation is higher than RTL_ALT. The default esteem for RTL_ALT is $15 \mathrm{~m}$.

\section{Loiter}

(GPS Dependent) Loiter Mode consequently endeavors to keep up the current area, heading and height. The pilot may fly the quad-rotor in loiter mode as though it was in manual. Discharging the sticks will keep on holding position.

\section{Auto}

In Auto mode, the AQR follows the predefined statement which was already stored in the ARDU autopilot that will help to navigate commands like to search the waypoint.

\section{ACCEPTABLE ViBRATION DAMPING FOR ALL MODES}

\section{A. Trimming}

Wind obviously has an in number impact on AQR and will push it around. Nonetheless, one might find that while flying in settles mode, even in a windless situation the copter has a tendency to dependably float away in the same heading. This can generally be rectified utilizing the "Save Trim" or 
"Programmed Trim" capacities (see Fig. 4).

\section{B. Setting Throttle Mid}

For checking the results of throttle mid position fly the copter in a stable mode for at least 30seconds to check, If the normal throttle is underneath 300 (i.e. 30\%) then, one has an exceptionally overwhelmed copter and it would be best to include some additional weight (i.e. A greater battery maybe) or diminishes the force in some other way (Smaller engines, move from a $4 \mathrm{~S}$ to a $3 \mathrm{~S}$ battery, and so forth).

In the event that the normal throttle is over 700 (i.e. 70\%) then one has an exceptionally underpowered copter. One ought to consider expanding the force of the engines and ESCs or utilize a higher voltage battery (i.e. Switch from a $3 \mathrm{~S}$ to a $4 \mathrm{~S}$ battery).

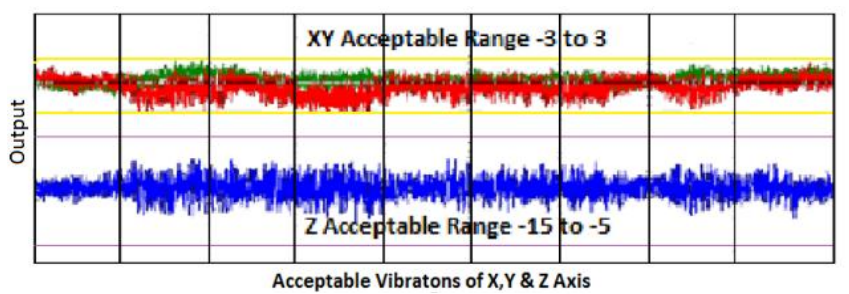

Fig 4 . Vibration range.

\section{ESC CALIBRATION AND CONTROL DOMAIN}

In Fig. 5, the ESC electronic speed controller controls the brushless DC motor and send the data to the receiver.

In Fig. 6, ESC moves throttle stick on top than connected to the battery pack for about 2 seconds. To confirm throttle, range the highest point a beep tone emitted.

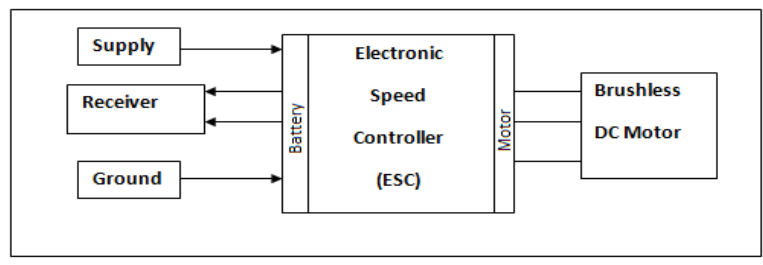

Fig. 5. ESC mode connection

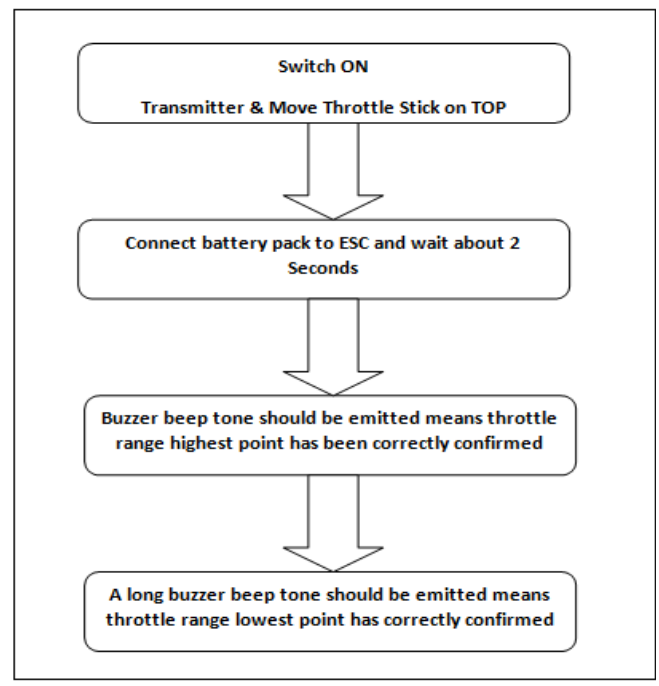

Fig. 6. ESC normal startup mode.

In Fig. 7, ESC throttle range setting is performed. Move throttle stick to bottom when the switch is on and connect to the battery pack by showing the buzzer. Several beeps indicate the quantity of Lithium Cells and on finishes the long buzzer tone emitted. Control domain and control flow diagram are shown in Fig. 8 and Fig. 9.

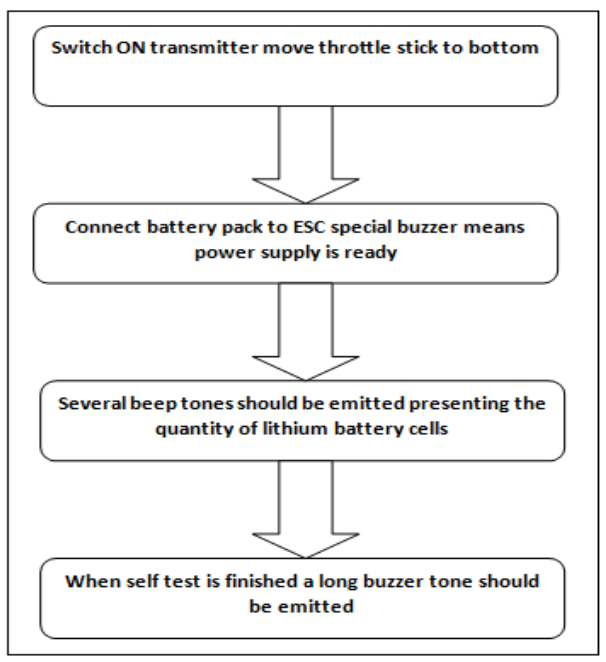

Fig. 7. ESC throttle range setting.

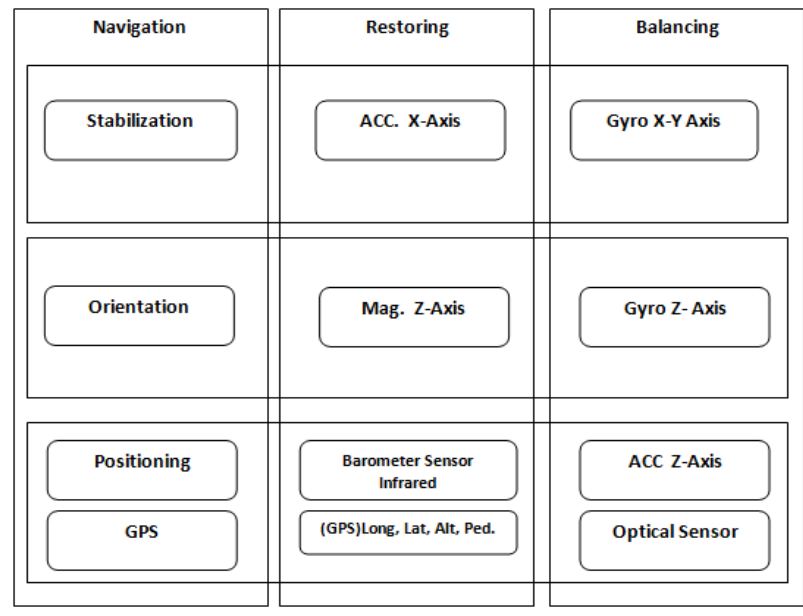

Fig. 8. Control functions, domain.

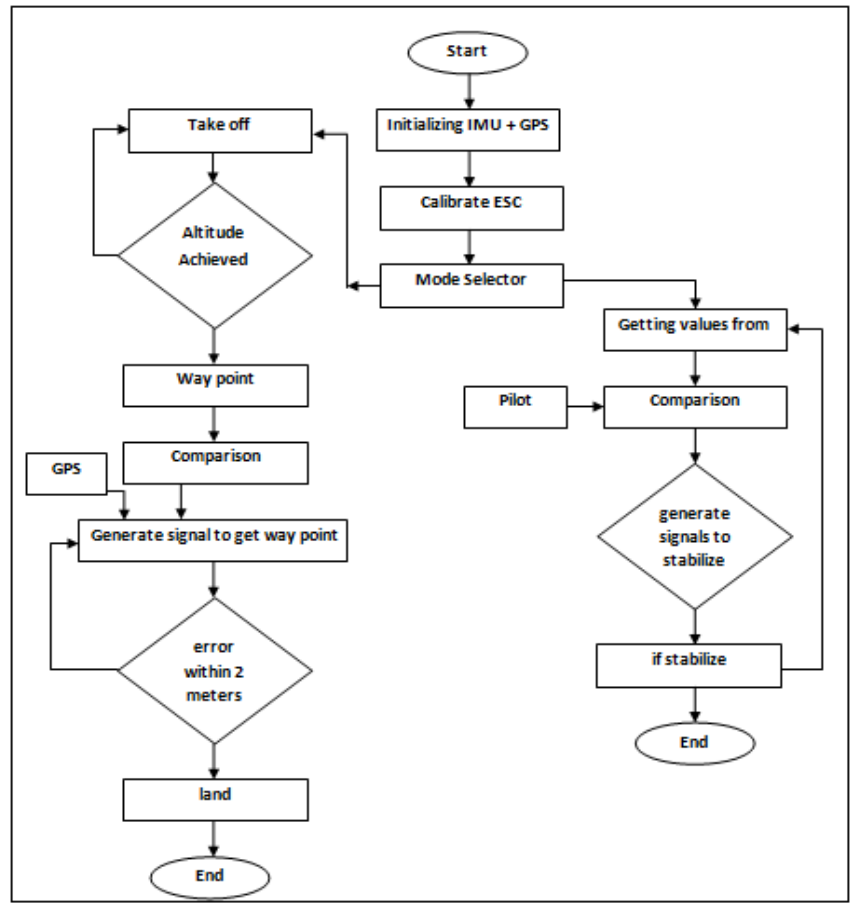

Fig. 9. Control flow diagram. 


\section{CONTROL FLOWCHART}

- Step A:

Plug in the battery, turn on the remote.

- Step B:

Initialize IMU and GPS. Wait until the IMU's (FC) and GPS are ready, you can see the LED status from Blinking to Stable state.

- Step C:

Mode Selection

If Mode 0:

- Step 1:

Getting the values from Gyro Accelerate and at the same time we are getting the Pilot's input.

- Step 2:

Comparing both the values.

- Step 3:

After comparing, signals generated to stabilize and go to that desired location or state or position.

- Step 4:

If Copter is stabilized and flying beautifully then ends the command otherwise feedback the values of sensors to start the process again.

If Mode 1:

\section{- Step 1:}

Auto Mode Active

Take Off command active, in which we set the altitude value (The vehicle will climb straight up from its current area to the elevation determined (in meters). This ought to be the first command of almost all missions. In the event that the mission is started while the copter is flying, the vehicle will climb straight up to the predetermined height, if the vehicle is as of now over the predefined elevation the takeoff order will be overlooked and the mission will move onto the following summon instantly.)

- Step 2:

If Altitude achieved.

Then move to step 3 otherwise repeat step 1 .

- Step 3 \& 4:

\section{Waypoint command}

(The vehicle will fly a straight line to the area determined as a lat, lon and height (in meters)) In this step we are comparing the values of GPS, location and waypoint values, so it can send signals to the Ardupilot where copter should move and in which direction.

- Step 5:

The GPS itself has 5 6-meter error, but it depends upon the value of HDOP and no of satellites, in this step we calculate the error between the desired locations. We make a circle from the desired location if the error value lies in the circle, then move to the next step which is Land command.

NOTE: You can also use the Delay command between Takeoff, Waypoint \& land commands.

\section{ESC CALIBRATION STEPS FOR ARDU-COPTER}

1) Turn Transmitter $\mathrm{ON}$ and set Throttle to the maximum.

2) Associate the Li-PO battery. The Ardupilot's red, blue and yellow LEDs will light up in a cyclical example. This implies that prepared to go into ESC adjustment.

3) With the transmitter throttle stick still high, separate and reconnect the battery

4) Hold up for ESCs to discharge the musical tone, the customary number of beeps showing your battery's cell check (i.e. 3 for $3 \mathrm{~S}, 4$ for $4 \mathrm{~S}$ ) and afterward an extra two beeps to demonstrate that the greatest throttle has been caught.

5) Now set Throttle to a minimum.

6) The ESCs ought to then discharge a long tone, demonstrating that the minimum throttle has been caught and the adjustment is finished.

7) In the event that the long tone, demonstrating fruitful adjustment was listening, the ESCs is "live" now and on the off chance that raise the throttle a bit they ought to turn. Test that the engines turn by raising the throttle a bit and afterward bringing down it once more.

8) Set the throttle to least and separate the battery to exit ESC-adjustment mode.

\section{CONTROl Diagram}

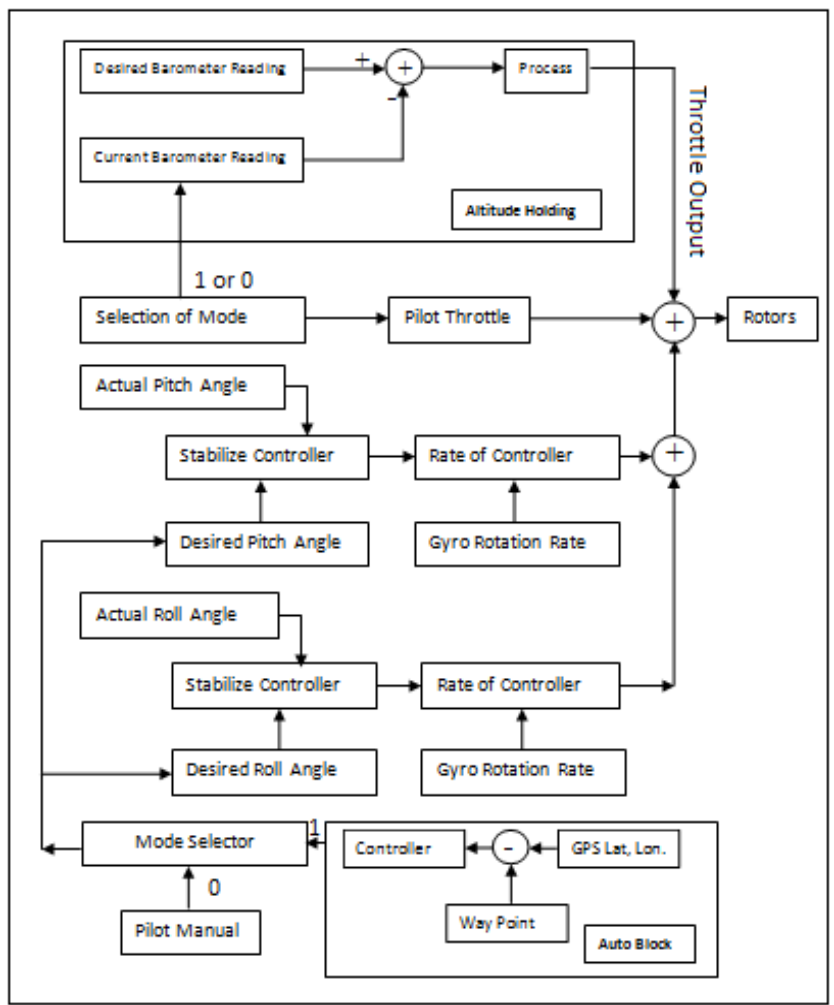

Fig. 10. Control diagram.

In the above Fig. 10 control diagram of Quad-Copter there are two blocks:

1) Auto Block

2) Altitude Hold Block

First, we have to understand what Auto mode in quad-rotor is.

In the AUTO block, pre-programmed navigation commands/way points saved in Ardupilot, but it is totally depending upon the Mode selection block which can be done by various methods. In this case Mode Selection is done by the TX itself by the pilot. 
After Arming the Copter it depends on the pilot if the pilot wants to take a regular stabilize flight by himself, there will be no signal transmitted through Mode Selection Channel, but if he wants to make an autonomous flight, mode selection channel is now High 1 or simply changed from the previous state.

\section{Automatic InITIALIZING CONDITIONS}

Initially the vehicle is in ground position and the controller must assure the throttle is down, secondly, it will change into an auto flight mode after that throttle elevates within a minute the throttle is heaved more than zero the vehicle starts the mission. From the beginning of the mission flight mode switches on the auto mode. If the mission statement changes, it sends the AQR above the impersonation instruction altitude and the take-off call will be completed. The vehicle will be in moving on the desired destination. When we want to control the vehicle manually convert it into the flight mode and switch it to alternate the flight mode like loiter or stabilize. Moreover, when we shift it back on the auto mode again than the mission statement will again restart from the initial charge. Throughout the task manually move about throttle and pitch inputs are ignored at that time yaw can be dominated with the yaw stick. That will permit for the sudden point the head of the vehicle has a camera on it as the vehicle flies on the mission. The autopilot will undertake the yaw governed as the AQR passes to the destination.

After selecting the mode and starting the mission Stabilize and Rate PID (Desired Angle and Rotation Rate) starts doing their magic to stabilize the copter and error rate.

Now, the next block is on Alt hold block, which is the mother of Loiter (Position) Mode without Alt hold mode Copter cannot be in Loiter Mode and remember that auto mode depends on these two modes. GPS (HDOP specially, GPS positioning and HDOP depend on No of Satellites).

The same Mode Selection steps for this block, but we know ALT Hold depend upon Barometer. After selecting the mode there is an error calculation of desired and current reading of the barometer, then the Throttle is finally set to maintain a fixed height (see Fig. 11 and Fig. 12).

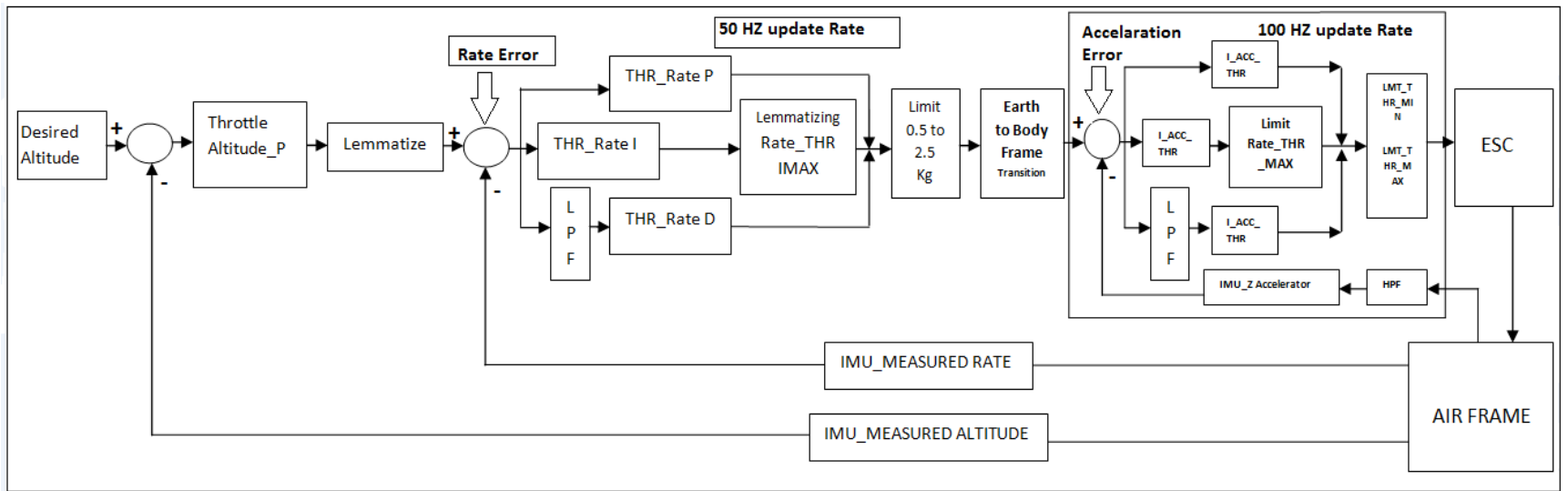

Fig. 11. Altitude hold block diagram.

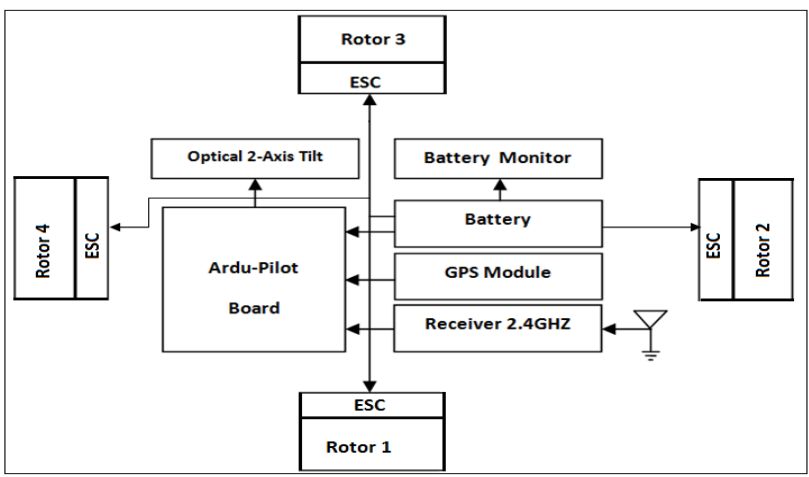

Fig. 12. Electrical wiring diagram.

\section{CONCLUSION}

The design of Autonomous Quad Rotor using Ardu-Pilot is really a challengeable task in which Electronic Speed Controller (ESC) is used. This venture has an extensive variety of utilization and covers all zone of electronic building blocks like installed controller control of BLDC engine correspondence. The results show that the response of this copter is much faster than other typical copter due to its instant reacting commands and switching parameters as shown in Fig. 13.

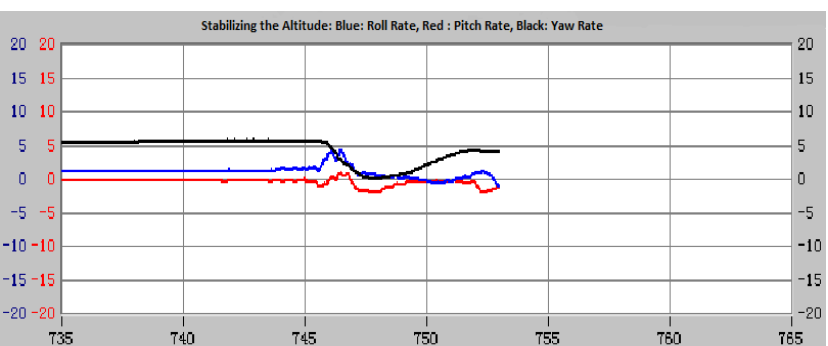

Fig.13(a). Pitch, yaw, roll before controlling.

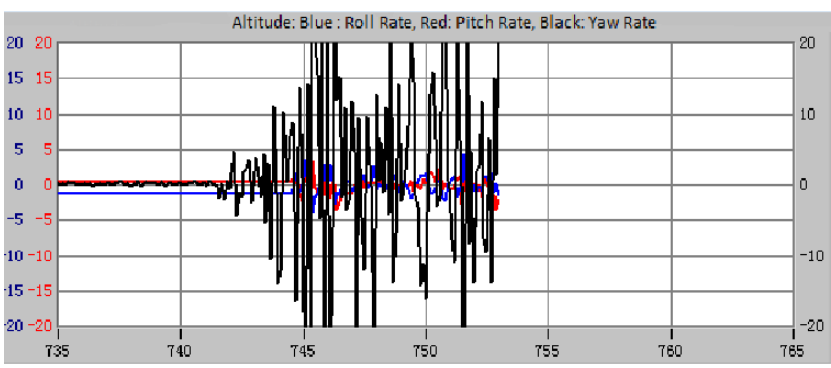

Fig. 13(b). Pitch, yaw, roll after controlling.

\section{ACKNOWLEDGMENT}

This work was support by Key Lab of College of Automation Engineering, Nanjing University of Aeronautics and Astronautics. 


\section{REFERENCES}

[1] S. Bouabdallah, A. Noth, and R. Siegwart, "PID vs LQ control techniques applied to an indoor micro quadrotor," in Proc. 2004 IEEE/RSJ International Conference on Intelligent Robots and Systems, 2004, vol. 3, pp. 2451-2456.

[2] S. Bouabdallah and R. Siegwart, "Backstepping and sliding-mode techniques applied to an indoor micro quadrotor," in Proc. the 2005 IEEE International Conference on Robotics and Automation, pp. 2247-2252.

[3] S. Bouabdallah, P. Murrieri, and R. Siegwart, "Design and control of an indoor micro quadrotor," in Proc. 2004 IEEE International Conference on Robotics and Automation, 2004, vol. 5, pp. 4393-4398.

[4] S. Bouabdallah and R. Siegwart, "Full control of a quadrotor," in Proc. International Conference on Intelligent Robots and Systems, 2007.

[5] E. H. Zheng and J. J. Xiong, "Quad-rotor unmanned helicopter control via novel robust terminal sliding mode controller and underactuated system, sliding mode controller," International Journal for Light and Electron Optics, vol. 125, no. 12, pp. 2817-2825, 2014.

[6] Z. Öznalbant and M. S. Kavsaoglu, "Design and flight test study of a VTOL UAV," in Proc. 53rd AIAA Aerospace Sciences Meeting, 2015.

[7] FAA Modernization and Reform Act of 2012. [Online]. Available: http://www.thequadcopterguy.blogspot.sg

[8] D. B. Wang, "Backstepping based nonlinear flight control strategy for 6 DOF aerial robot," in Proc. 2008 International Conference on Smart Manufacturing Application.

[9] Z. A. Ali, D. B. Wang, M. Aamir, "Design a robust RST controller for stabilization of a tri-copter UAV," Pakistan Journal of Engineering Technology \& Science, vol. 5, issue 1, June 2015.

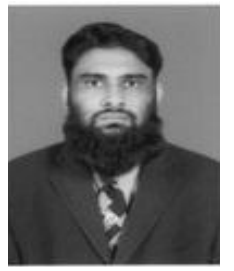

Rana Javed Masood received the ME degree of industrial control and automation from Hamdard University of Engineering and Technology, Pakistan Currently, he is a Ph.D. student in control and automation engineering from Nanjing University of Aeronautics and Astronautics, China. He has been with Usman Institute of Engineering and Technology, since August 2010. His research interests focus on virtual reference feedback tuning, data driven control, MFA control, and UAV flight control.

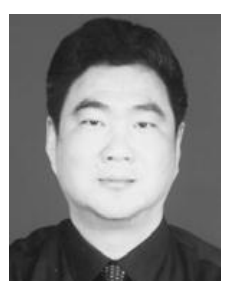

Dao Bo Wang is a professor in the College of Automation Engineering at Nanjing University of Aeronautics and Astronautics, China. He was an official designated visiting scholar at University of Bath, UK from 1987 to 1989 and senior visiting scholar at University of Waterloo, Ontario, Canada in 1999. His research interests include unmanned aerial vehicle flight control and aeronautical simulation.

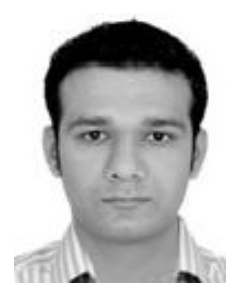

Zain Anwar Ali received the B.S. degree in electronic engineering from the Sir Syed University of Engineering and Technology, Karachi, Pakistan in 2010. He is currently doing his $\mathrm{PhD}$ in control theory and its automation at Nanjing University of Aeronautics \& Astronautics, Nanjing, China since 2015. He is working as a faculty member and researcher in the Department of Electronic Engineering, Sir Syed University of Engineering and

Technology.

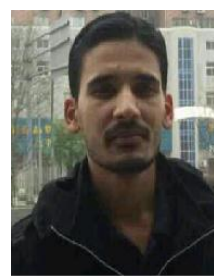

Suhaib Masroor received the B.S. degree in electronic engineering from Sir Syed University of Engineering and Technology, Karachi, Pakistan in 2008. Currently he is doing his Ph.D. in control engineering at Shanghai University.

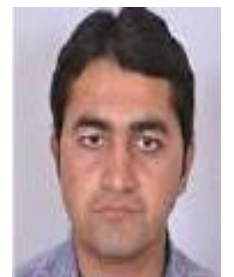

Technology.
Muhammad Shafiq received the B.S. degree in electronic engineering from Sir Syed University of Engineering and Technology, Karachi, Pakistan in 2011. Now, he is pursuing his M.S. degree in telecommunication engineering from Sir Syed University of Engineering and Technology, Karachi, Pakistan. He is working as a faculty member and researcher in the Department of Electronic Engineering, Sir Syed University of Engineering and 